\title{
Validación de escala
}

\section{de la seguridad alimentaria doméstica en Antioquia, Colombia}

\author{
Marta Cecilia Álvarez, M en Des Soc y Educ,(1) Alejandro Estrada, M en Epidemiol,(2) \\ Elizabeth Cristina Montoya, ND, (1) Hugo Melgar-Quiñónez, PhD.(3)
}

\section{Álvarez MC, Estrada A, Montoya EC, Melgar-Quiñónez H. Validación de escala de la seguridad alimentaria doméstica en Antioquia, Colombia. Salud Publica Mex 2006;48:474-48I.}

\section{Resumen}

Objetivo. Adaptar y validar en hogares de Antioquia, Colombia, una escala de seguridad alimentaria anteriormente aplicada en hogares de Caracas, Venezuela. Material y métodos. El estudio se realizó en 44 municipios del departamento de Antioquia, Colombia, durante los años 2003 y 2004, en una muestra representativa de los hogares rurales y urbanos del departamento de Antioquia, constituida por I 624 hogares con niños menores de 10 años; los hogares fueron seleccionados de manera aleatoria, con un índice de confianza de $95 \%$ y un error de $3 \%$. Se validó la escala de seguridad alimentaria doméstica utilizada por Paulina Lorenzana en Venezuela, y se determinó la consistencia interna de la escala mediante el coeficiente de correlación de Spearman y el coeficiente alfa de Cronbach. La validez del constructo se estableció mediante el método de componentes principales para datos categóricos. Se utilizaron el procedimiento de Prinqual y el modelo de Rasch para definir los componentes y los ítems de la escala. Resultados. El análisis factorial arrojó dos componentes: I) las variables relacionadas con "inseguridad alimentaria sin hambre" que lo explican en $95 \%$ y 2 ) las relacionadas con "inseguridad alimentaria con hambre" que lo explican en $89.4 \%$. El coeficiente alfa de Cronbach para el primer componente fue de de 0.95 y para el segundo de 0.89 . Al ser analizados con el modelo Rasch, todos los ítems presentaron valores infit en un rango de 0.8 a I.2. La escala se correlacionó significativa-
Álvarez MC, Estrada A, Montoya EC, Melgar-Quiñónez H. Validation of a household food security scale in Antioquia, Colombia.

Salud Publica Mex 2006;48:474-48I.

\section{Abstract}

Objective. To adapt and validate in households of Antioquia, Colombia, a food security scale previously applied in households of Caracas,Venezuela. Material and Methods. The study was carried out in 44 municipalities in the department of Antioquia, Colombia, in 2003 and 2004, with a randomly selected sample of I 624 rural and urban households with children under 10 years of age, representative for family units located in the department of Antioquia. The sample was selected using a confidence interval of $95 \%$ and an error of $3 \%$. Household food security scale previously used by Paulina Lorenzana in Venezuela were validated for this survey. Internal consistency of the scale was determined using the Spearman correlation coefficient and Cronbach's Alpha coefficient. Construct validity was established through principal components analysis for categorical data. Prinqual procedure and Rasch modeling were used to define the components and items in the scale. Results. Factor analysis showed two components: I) variables related to "food insecurity without hunger", which is explained in 95\%; 2) variables related to "food insecurity with hunger", which is explained in $89.4 \%$. Cronbach's Alpha coefficients for "food insecurity without hunger" and "food insecurity with hunger" were 0.95 and 0.89 , respectively. When analyzed using Rasch modeling, all items showed infit values within a range of 0.8 and 1.2. The scale correlated significantly $(p<0.000)$ with food availability, begging, children's labor, household size,

Este estudio fue financiado por la Gobernación de Antioquia, la Dirección Seccional de Salud de Antioquia y el Plan de Mejoramiento Alimentario y Nutricional de Antioquía (MANA).

(I) Grupo de Investigación en Alimentación y Nutrición Humana, Escuela de Nutrición y Dietética, Universidad de Antioquia. Colombia.

(2) Grupo de Demografía y Salud, Facultad Nacional de Salud Pública. Escuela de Nutrición y Dietética, Universidad de Antioquia. Colombia.

(3) Departamento de Nutrición Humana, The Ohio State University. EUA.

Fecha de recibido: 20 de febrero de 2006 - Fecha de aprobado: 28 de julio de 2006 Solicitud de sobretiros: Marta Cecilia Álvarez. Escuela de Nutrición y Dietética, Universidad de Antioquia, Colombia. Grupo de Investigación en Alimentación y Nutrición Humana. Carrera 75 Nº 65-87. Ciudadela de Robledo. Universidad de Antioquia. Correo electrónico: mcau@pijaos.udea.edu.co 
mente con la disponibilidad de alimentos, el recurso de la limosna, el trabajo infantil, el tamaño del hogar y la ocupación del jefe de familia $(p<0.000)$. Conclusión. La escala puede considerarse un instrumento confiable para la valoración de la inseguridad alimentaria en los hogares de Antioquia.

Palabras clave: validación; inseguridad alimentaria; Antioquia; Colombia and occupation of the head of household. Conclusions. The scale can be considered a reliable instrument for assessing food insecurity in Antioquia households.

Key words: validation; food insecurity; Antioquia; Colombia
$\mathrm{L}$ a importancia de la seguridad alimentaria y nutricional en los hogares ha sido abordada por diferentes organismos internacionales y nacionales, ${ }^{1}$ y los gobiernos de los países participantes se han comprometido a garantizarle a la población el acceso a la cantidad y calidad de los alimentos necesarios para llevar una vida sana y productiva, así como reducir el hambre y la desnutrición. Sin embargo, la Organización de las Naciones Unidas para la Agricultura y la Alimentación (FAO) estimó que en el periodo 2000-2002 "había en todo el mundo 852 millones de personas desnutridas; de estas, 815 millones vivían en los países en desarrollo, 28 millones en países en transición y nueve millones en países desarrollados." ${ }^{2}$ Este mismo organismo reportó que en los países en desarrollo, el número de personas desnutridas se redujo sólo en nueve millones durante la década posterior a la Cumbre Mundial sobre Alimentación. ${ }^{2}$

Las estadísticas de desnutrición son definidas por la FAO, con base en la disponibilidad per cápita de energía obtenida de las hojas de balance de alimentos de cada país y con la estimación de las necesidades mínimas alimentarias per cápita. ${ }^{3}$ Estas cifras son útiles para evaluar la suficiencia alimentaria nacional, aunque no informan sobre la distribución de los alimentos y el acceso a ellos por parte de hogares e individuos, ${ }^{4}$ ni permiten conocer la magnitud del hambre como la expresión más extrema de inseguridad alimentaria.

Para evaluar la magnitud y gravedad de la desnutrición, se han utilizado las encuestas sobre ingresos y gastos de los hogares, las hojas de balance de alimentos, las encuestas sobre consumo de alimentos y las encuestas antropométricas. ${ }^{4}$ En los años noventa, en Estados Unidos, ante la necesidad de conocer de manera directa el grado de seguridad alimentaria en los hogares, se introdujo una escala para medirla, la cual se originó en la escala conocida como de Radimer/ Cornell y la escala para la identificación del hambre en la infancia., ${ }^{5,6}$ La evidencia para su construcción se obtuvo de las entrevistas a profundidad realizadas por
Radimer a mujeres que vivían en zonas rurales, que arrojaron los siguientes resultados: a) la inseguridad alimentaria se experimenta de manera diferente en los niños y en los adultos; b) el evento tiene cuatro componentes: calidad, cantidad, seguridad y aceptabilidad, y c) la inseguridad alimentaria con hambre representa el nivel más severo del fenómeno. ${ }^{7}$ Con base en los estudios anteriores, desde 1995, se introdujo en Estados Unidos el módulo de Inseguridad Alimentaria en la Encuesta Nacional de Población (CPS, por sus siglas en inglés). ${ }^{8}$

En América Latina, Lorenzana y Sanjur modificaron y validaron el índice de hambre desarrollado en Estados Unidos por Wehler y colaboradores en 1992, ${ }^{6}$ para aplicarlo a 1999 hogares pobres de Caracas. ${ }^{9}$ Por su parte, Melgar-Quiñónez y su grupo estudiaron la percepción y experiencia de inseguridad alimentaria en la población latina de California (EUA) e interpretaron los ítems incluidos en la escala mediante la técnica de grupos focales; ${ }^{10}$ más adelante, realizaron estudios de validación en áreas rurales mexicanas. ${ }^{11} \mathrm{En}$ Argentina, en 2003, la Oficina del Banco Mundial aplicó la escala del Departamento de Agricultura de Estados Unidos (USDA), ${ }^{12}$ la que finalmente fue adaptada y validada en Brasil por Pérez-Escamilla y un grupo de investigadores de la universidad de Campinas. ${ }^{13}$

En Colombia, y en el departamento de Antioquia en particular, no se contaba con un instrumento adaptado y validado para evaluar de manera sencilla y rápida la seguridad alimentaria en el hogar. Esta situación representaba una limitación muy importante para estimar la magnitud y gravedad del problema, y darle seguimiento a las metas del milenio, suscritas por el gobierno de Colombia. Es por ello que la Gobernación del Departamento de Antioquia y el Plan de Mejoramiento Alimentario y Nutricional (MANA), apoyaron la adaptación y validación de la escala previamente desarrollada en Venezuela ${ }^{9}$, ya que ésta fue aplicada en un contexto geográfico y socioeconómico similar al de los hogares antioqueños. 
El objetivo del presente estudio fue adaptar y validar en hogares de Antioquia, Colombia, la escala de seguridad alimentaria aplicada en hogares de Caracas, Venezuela.

\section{Material y métodos}

Previo a la aplicación de la escala de seguridad alimentaria doméstica ajustada y validada por Lorenzana en Caracas, Venezuela, ${ }^{9}$ se ajustó la redacción de los ítems mediante grupos focales (cuadro I). La escala estaba constituida por 12 ítems relacionados con la disponibilidad de dinero para la compra de alimentos, la disminución de comidas en el hogar por falta de dinero y la experiencia de hambre de alguno de los integrantes del hogar (cuadro I). La frecuencia de ocurrencia de cada ítem se codificó de la siguiente manera: tres veces como "siempre"; dos, "a veces"; una, "rara vez", y cero, "nunca". Esta codificación permitió generar un puntaje de inseguridad alimentaria con un rango entre 0 y 36 puntos, a partir del cual los hogares se clasificaron en cuatro categorías: 0 puntos como "seguros"; 1-12, "inseguridad alimentaria leve"; 13-24, "inseguridad alimentaria con hambre moderada", y 25 o más, "inseguridad alimentaria con hambre severa".

Se realizó un estudio descriptivo transversal, con una muestra bietápica estratificada. En una primera etapa, se estratificaron los 125 municipios del departamento de acuerdo con los rangos de pobreza, así: $>90 \%$ (I), $70-90 \%$ (II), $50-<70 \%$ (III) y $<50 \%$ (IV), para caracterizar el perfil alimentario y nutricional de los hoga- res de las regiones de Antioquia. El número de municipios se definió con una confianza de $95 \%$ y un error de $10 \%$, para obtener una muestra final de 44 . Cuando en una categoría sólo se encontró un municipio, se incluyó.

En una segunda etapa se seleccionaron los hogares; la muestra fue de 2520 y contó con una confianza de $95 \%$, una proporción del evento en la población de $50 \%$ y un error de muestreo de $3 \%$; se aplicó un efecto del diseño de 2 y un sobremuestreo de $20 \%$ para recuperar posibles caídas (pérdidas) en la información. La selección de la muestra correspondió a cada uno de los estratos de las nueve regiones administrativas del departamento de Antioquia y la ciudad de Medellín. La selección final del hogar se realizó de forma aleatoria, con el apoyo de la cartografía de cada municipio, suministrada por el Departamento Administrativo Nacional de Estadística de Colombia (DANE). De manera aleatoria, se seleccionaron las secciones, manzanas y viviendas de los sectores. Para la zona rural, se establecieron dos conglomerados de veredas de acuerdo con su calidad de vida, y de manera aleatoria se seleccionó una vereda de cada estrato. Esta muestra final de 2520 incluyó hogares con niños menores de 10 años.

Con el fin de adaptar y validar la escala de seguridad alimentaria doméstica, se aplicó en hogares que tuvieran niños menores de 10 años, lo que dio una muestra total de 1624 hogares distribuidos en las nueve regiones administrativas y Medellín. De esos hogares, $52.7 \%$ se encuentra en el área urbana y $47.3 \%$ en la rural. Previa capacitación y estandarización, nutricio-

\section{Cuadro I \\ Escala de seguridad alimentaria en el hogar aplicada en Antioquia, Colombia (2004-2005)}

En los últimos seis meses, ¿con qué frecuencia se presentaron las siguientes situaciones en su hogar?

Puede responderme con: $S=$ siempre; $A V=a$ veces; $R V=$ rara vez

\begin{tabular}{|c|c|c|c|c|c|}
\hline \multirow[t]{2}{*}{ Items } & \multirow[b]{2}{*}{ Sí } & \multirow[b]{2}{*}{ No } & \multicolumn{3}{|c|}{ Frecuencia } \\
\hline & & & $S$ & $A V$ & $R V$ \\
\hline I. Faltó dinero en el hogar para comprar alimentos & & & 3 & 2 & I \\
\hline 2. Alguna persona adulta del hogar comió menos de lo que desea por falta de dinero para comprar alimentos & & & 3 & 2 & I \\
\hline $\begin{array}{l}\text { 3. En el hogar se disminuyó el número de comidas usuales, como dejar de desayunar, almorzar o comer por falta } \\
\text { de dinero para comprar alimentos }\end{array}$ & & & 3 & 2 & I \\
\hline 4. Algún adulto dejó de desayunar, almorzar o comer por falta de dinero para comprar alimentos & & & 3 & 2 & I \\
\hline 5. Algún adulto comió menos en la comida principal porque la comida no alcanzó para todos & & & 3 & 2 & I \\
\hline 6. Algún adulto se quejó de hambre por falta de alimentos en el hogar & & & 3 & 2 & I \\
\hline 7. Algún adulto se acostó con hambre porque no alcanzó el dinero para la comida & & & 3 & 2 & I \\
\hline 8. Se compraron menos alimentos indispensables para los niños porque el dinero no alcanzó & & & 3 & 2 & I \\
\hline 9. Algún niño dejó de desayunar, almorzar o comer por falta de dinero para comprar alimentos & & & 3 & 2 & I \\
\hline 10. Algún niño comió menos en la comida principal porque la comida no alcanzó para todos & & & 3 & 2 & 1 \\
\hline II. Algún niño se quejó de hambre por falta de alimentos en el hogar & & & 3 & 2 & I \\
\hline 12. Algún niño se acostó con hambre porque no alcanzó dinero para la comida & & & 3 & 2 & I \\
\hline
\end{tabular}


nistas dietistas aplicaron la escala, y entrevistaron a la madre o a la persona encargada de la preparación de alimentos en el hogar. Para la entrevista, se partió de la premisa de que se hubieran presentando las situaciones descritas en los ítems, en los últimos seis meses por falta de dinero.

Para determinar la diversidad de alimentos disponibles en el hogar, se llevó a cabo la sumatoria del número de alimentos diferentes de que se dispuso, durante la semana anterior a la entrevista. Esta variable se relacionó con la puntuación de escala, dado que la variabilidad de la alimentación es un indicador de su calidad y de la seguridad alimentaria.

\section{Análisis de datos}

Para llevar a cabo el análisis estadístico de los datos se utilizó SPSS versión 10, y para determinar el ajuste de los datos obtenidos al modelo Rasch, ${ }^{14}$ el software Winsteps. Se estableció la consistencia interna de la escala con el coeficiente de correlación y el alfa de Cronbach. La validez del constructo se estimó con el método de componentes principales para datos categóricos. El procedimiento de Prinqual ${ }^{15}$ y el modelo de Rasch $^{14}$ se utilizaron para definir los componentes y los ítems de la escala. Finalmente, se utilizó la correlación de Spearman, ji cuadrada y las pruebas no paramétricas de Kruskall Wallis y U de Mann Whitney para determinar la relación de la escala con otras variables asociadas con el fenómeno de inseguridad alimentaria.

\section{Consentimiento informado}

Esta investigación fue aprobada por el comité de ética del Área de la Salud de la Universidad de Antioquia-Medellín, Colombia. Los jefes de los hogares participantes firmaron el consentimiento informado, el cual incluyó los principios éticos para las investigaciones médicas en seres humanos de la declaración de Helsinki.

\section{Resultados}

\section{Adaptación de la escala}

Las participantes en los grupos focales coincidieron en señalar que, por falta de dinero en el hogar, se realizaban estrategias de ajuste alimentario similares a las que se enunciaban en los ítems de la escala de Venezuela. Además, agregaron algunas estrategias negativas como pedir limosna, hacer trabajar a los niños, y adoptar una actitud de resignación. Los ítems de la escala fueron comprendidos por las mujeres participantes, aunque fue necesario redactar algunos de ellos con términos comúnmente utilizados en Colombia para tener mayor precisión del aspecto que deseaba evaluarse.

\section{Consistencia interna de la escala}

\section{Análisis factorial}

Se encontraron correlaciones altas y significativas entre las pruebas que conforman la escala de percepción, que indicaban posibles componentes o factores subyacentes en su interior (cuadro II). El análisis factorial exploratorio a través del método de componentes principales para datos categóricos que utiliza el procedimiento Prinqual arrojó dos componentes que explicaban un alto porcentaje de la variabilidad de la inseguridad alimentaria. Las variables relacionadas con la "inseguridad alimentaria sin hambre" explican en 95\% su comportamiento, mientras que las variables relacionadas con la "inseguridad alimentaria con hambre" lo explican en $89.4 \%$. Para la escala en su totalidad la variabilidad se explica en 99 por ciento.

Las variables que cargan principalmente cada componente se muestran en el cuadro III; las que se encuentran subrayadas son las que están cargando al correspondiente componente y no al otro (cuadro III). En el componente "inseguridad alimentaria sin hambre" se correlacionaron tres ítems: a) ingesta menor de alimentos por los adultos; $b$ ) menor compra de alimentos para los niños, y c) falta de dinero para la compra de alimentos. Para el componente inseguridad alimentaria con hambre, se correlacionaron nueve ítems en los que se incluyen todos los relacionados con hambre y con la disminución de las comidas por los adultos y los niños (cuadro III).

A partir de las correlaciones establecidas, se observa cómo los ítems 1, 2 y 8 presentan alta reciprocidad entre sí y baja en relación con los demás ítems de la escala. Por ello, el componente de "inseguridad alimentaria sin hambre" quedó conformado por estos ítems con sus respectivas cargas factoriales (cuadros II y III). Una situación semejante se observa al analizar las correlaciones del ítem 12 con los otros, con excepción de 1,2 y 8, con los que tiene una baja correspondencia. Por consiguiente, los nueve ítems 3-7 y 9-12 integran el componente de "inseguridad alimentaria con hambre" con sus respectivas cargas factoriales (cuadros II y III).

\section{Modelo de Rasch}

Los resultados obtenidos al aplicar el modelo Rasch permitieron poner en evidencia el buen ajuste que 


\section{Cuadro II \\ Matriz de correlaciones de la escala de la seguridad alimentaria doméstica aplicada en Antioquia, Colombia (2004-2005)}

\begin{tabular}{|c|c|c|c|c|c|c|c|c|c|c|c|c|}
\hline \multirow[b]{2}{*}{ Ítems de la escala } & \multicolumn{12}{|c|}{ Ítems de la escala } \\
\hline & P7 & PII & P6 & PIO & Pq & P5 & P4 & P3 & P2 & P8 & $P I$ & $P / 2$ \\
\hline P7 & 1 & 0.787 & 0.815 & 0.56 & 0.469 & 0.467 & 0.448 & 0.396 & 0.385 & 0.226 & 0.23 & 0.915 \\
\hline PII & & I & 0.908 & 0.58 & 0.49 & 0.495 & 0.46 & $0.4 \mathrm{II}$ & $0.4 I$ & $0.26 \mathrm{I}$ & 0.273 & $0.79 \mid$ \\
\hline P6 & & & 1 & 0.573 & 0.495 & 0.508 & 0.483 & 0.442 & 0.412 & 0.259 & 0.263 & 0.794 \\
\hline PIO & & & & I & 0.775 & 0.8 & 0.656 & 0.617 & 0.514 & 0.305 & 0.301 & 0.601 \\
\hline P9 & & & & & 1 & 0.707 & 0.817 & 0.748 & 0.518 & 0.3 & 0.299 & 0.509 \\
\hline P5 & & & & & & I & 0.717 & 0.654 & 0.58 & 0.355 & 0.343 & 0.484 \\
\hline P4 & & & & & & & 1 & 0.852 & 0.573 & 0.349 & 0.334 & 0.465 \\
\hline P3 & & & & & & & & 1 & 0.577 & 0.383 & 0.36 & 0.414 \\
\hline $\mathrm{P} 2$ & & & & & & & & & 1 & 0.607 & 0.588 & 0.38 \\
\hline P8 & & & & & & & & & & 1 & 0.814 & 0.216 \\
\hline $\mathrm{PI}$ & & & & & & & & & & & I & 0.221 \\
\hline
\end{tabular}

Cuadro III

Estructura factorial, MATRIZ DE CARGAS FACTORIALES DE LA ESCALA DE LA SEGURIDAD ALIMENTARIA DOMÉSTICA aplicada en Antioquia, Colombia (2004-2005)

\begin{tabular}{|c|c|c|c|}
\hline \multirow[b]{2}{*}{ Núm. } & \multirow[b]{2}{*}{ Ítems } & \multicolumn{2}{|c|}{ Componentes } \\
\hline & & $\begin{array}{l}\text { Inseguridad alimentaria } \\
\text { sin hambre }\end{array}$ & $\begin{array}{c}\text { Inseguridad alimentaria } \\
\text { con hambre }\end{array}$ \\
\hline I & Algún adulto se acostó con hambre porque no alcanzó el dinero para la comida & 0.41 & -0.60 \\
\hline 2 & Algún niño se quejó de hambre por falta de alimentos en el hogar & 0.44 & -0.61 \\
\hline 3 & Algún adulto se quejó de hambre por falta de alimentos en el hogar & 0.44 & -0.64 \\
\hline 4 & Algún niño comió menos en la comida principal porque la comida no alcanzó para todos & 0.52 & -0.67 \\
\hline 5 & Algún niño dejó de desayunar, almorzar o comer por falta de dinero para comprar alimentos & 0.52 & -0.64 \\
\hline 6 & Algún adulto comió menos en la comida principal porque la comida no alcanzó para todos & 0.53 & -0.66 \\
\hline 7 & Algún adulto se acostó con hambre porque no alcanzó el dinero para la comida & 0.54 & -0.66 \\
\hline \multirow[t]{2}{*}{8} & En el hogar se disminuyó el número de comidas usuales, como dejar de desayunar, almorzar & & \\
\hline & o comer por falta de dinero para comprar alimentos & 0.55 & -0.62 \\
\hline 9 & Alguna persona adulta del hogar comió menos de lo que desea por falta de dinero para compr & alimentos 0.81 & -0.25 \\
\hline 10 & Se compraron menos alimentos indispensables para los niños porque el dinero no alcanzó & 1.57 & 0.95 \\
\hline II & Falta dinero en el hogar para comprar alimentos & 1.55 & 0.96 \\
\hline 12 & Algún niño se acostó con hambre porque no alcanzó dinero para la comida & 0.44 & -0.59 \\
\hline
\end{tabular}

tienen los ítems evaluados, similar a lo planteado anteriormente en el análisis factorial, donde se generaron dos componentes o factores que explican la inseguridad alimentaria. Los valores de ajuste (infit) para cada uno de los ítems en el modelo Rasch se encuentran dentro del margen 0.8-1.2, lo cual indica un buen ajuste, debido a que lo ideal serían valores infit de 1; pero como regla, los valores entre 0.8 y 1.2 son generalmente aceptables; valores mayores serían inapropiados y menores de 0.8 se consideran sobreajustados. ${ }^{14}$ Así, el resultado del modelo de Rasch indica, además, la estrecha relación que existe entre los ítems de la escala, y corroboran la validez del instrumento (figura 1).

Debe tenerse en cuenta que el modelo de Rasch permitió igualmente determinar la confiabilidad de 
la escala, además de agrupar los ítems en dos componentes.

\section{Severidad secuencial}

A mayor severidad de la inseguridad alimentaria planteada por los ítems, se observó menor frecuencia de respuesta positiva, la cual se relaciona con la experiencia de hambre en el hogar (figura 2).

\section{Correlación de la escala con otras variables}

La correlación entre la diversidad de la disponibilidad de alimentos y la puntuación de la escala fue inversa y significativa $(r=-0.650, p<0.000)$. Además, se obtuvo una correspondencia significativa con las siguientes variables: a) actitud de resignación de parte de la mujer cabeza de hogar cuando no hay dinero para comprar alimentos $(r=0.74, p<0.000) ; b)$ recurso de la

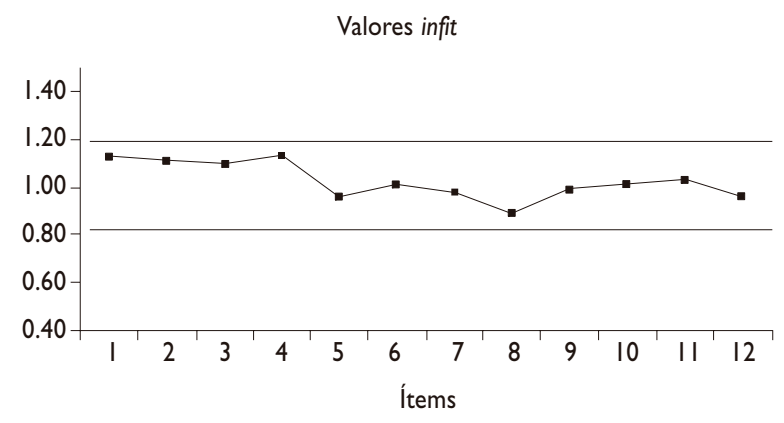

Figura I.Valores de los ítems

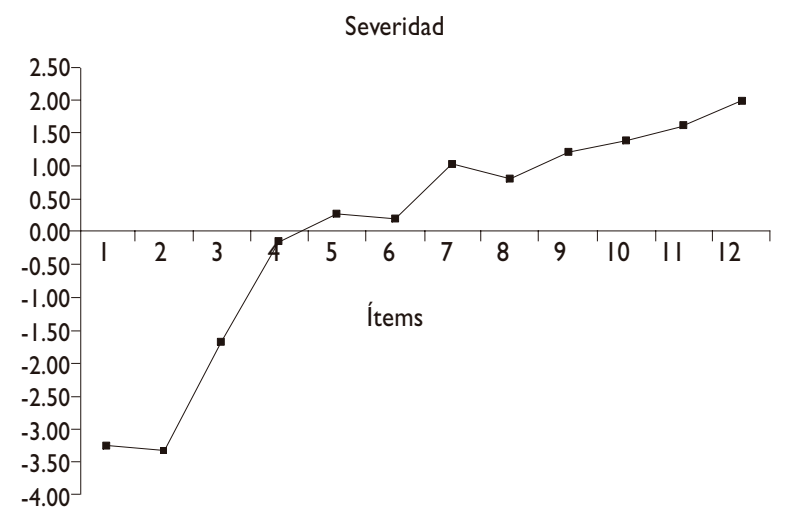

Figura 2. Severidad relativa de los ítems limosna cuando no hay alimentos por falta de dinero $(\mathrm{r}=0.64, p<0.000) ; \mathrm{c})$ trabajo de un menor de 10 años $(\mathrm{r}=0.31, p<0.000), \mathrm{y} d)$ número de personas que integran el hogar $(r=0.23, p<0.000)$. Finalmente, la escala se relacionó de forma significativa con la ocupación del padre, ocupación del jefe del hogar, la asistencia social alimentaria, tipología del hogar y lugar de residencia $(p<0,000)$. No se encontró asociación estadística entre tenencia de predios y producción de alimentos.

\section{Discusión}

La adaptación de la escala validada en hogares pobres de Caracas, Venezuela, ${ }^{9}$ fue una estrategia adecuada para definir la escala para Antioquia, Colombia, y perfilar su aplicación en el país. Por las similitudes geográficas, idiomáticas, sociales y culturales entre ambos países, las adaptaciones que se realizaron fueron muy pocas y básicamente consistieron en la adecuación de la redacción para que hubiera una mayor comprensión de cada uno de los ítems. La exploración sobre los ajustes alimentarios que realizan los hogares, cuando no tienen dinero suficiente para comprar alimentos, ratifican que ante esta situación los hogares en diferentes contextos sociodemográficos y culturales toman decisiones similares. $5,7,10,16$

Los alfas de Cronbach encontrados indican una alta homogeneidad y equivalencias de las respuestas. Se trata de una escala que posee un índice de consistencia interna satisfactorio, que puede considerarse un instrumento confiable para la valoración de la inseguridad alimentaria. Los alfas de Cronbach encontrados en este estudio fueron similares a los reportados por Lorenzana para el componente relacionado con modificaciones en el consumo de alimentos (0.91), para el componente sobre la experiencia de hambre $(0,88)$, y para la escala con los 12 ítems (0.92). ${ }^{16}$ Pérez-Escamilla obtuvo resultados similares al aplicar la escala de la USDA en Campiñas, Brasil, tanto en una muestra por conveniencia, como en una muestra representativa de esa zona $(0.91) \cdot{ }^{13}$ Los ítems se adhieren revelando dos componentes de la inseguridad alimentaria y confirmando los componentes teóricos del constructo de inseguridad alimentaria planteados por Frongillo. ${ }^{7}$

Los valores de ajuste (infit) para cada uno de los ítems en el modelo Rasch (0.8-1.2) indican un buen ajuste. Además, los ítems mostraron consistencia con la probabilidad de ocurrencia según el grado de severidad, de tal manera que aquellos relacionados con la situación más extrema de inseguridad alimentaria presentaron una menor frecuencia.

La severidad secuencial de la escala validada en Antioquia presenta una tendencia similar a la encon- 
trada por cinco encuestas al aplicar la escala de Radimer / Cornell, en las cuales se aprecia que los ítems de mayor severidad, por estar relacionados con la experiencia del hambre, presentan menores proporciones de respuestas positivas. ${ }^{7} \mathrm{Al}$ respecto, Frongillo concluye que la escala presenta una buena consistencia interna, ${ }^{7}$ dado que lo esperado es que una mayor proporción de hogares que no disponen de suficiente dinero para la compra de alimentos logren, con los ajustes alimentarios enunciados en los otros ítems, que sus miembros no experimenten la sensación de hambre, aun cuando la calidad de la dieta no sea la adecuada. ${ }^{8}$

La correlación del puntaje de inseguridad alimentaria (0-36 puntos) con la variedad de la disponibilidad de alimentos en hogar fue inversa $(r=-0.65, p<0.01)$ y mayor a la encontrada en los hogares pobres de Caracas $(\mathrm{r}=-0.48, p<0.01) .{ }^{17} \mathrm{La}$ diversidad de alimentos disponibles en el hogar, entendida como el número de alimentos diferentes disponibles para un periodo, ${ }^{18}$ es un indicador que se relaciona significativamente con la inseguridad alimentaria doméstica: a menor disponibilidad de alimentos en el hogar, mayor riesgo de inseguridad alimentaria. Pérez-Escamilla, en Brasil, y Melgar-Quiñónez, en México, reportaron que el nivel de severidad de la inseguridad alimentaria presentaba asociación negativa con la probabilidad del consumo diario de frutas, vegetales y alimentos de origen animal. ${ }^{11,13}$

La correlación estadísticamente significativa del puntaje de inseguridad alimentaria con estrategias negativas que los hogares realizan para enfrentar la inseguridad alimentaria, tales como pedir limosna y el trabajo infantil, corroboran la utilidad de la escala para detectar situaciones alimentarias extremas. La escala también se correlacionó con el número de personas que integran el hogar, lo cual es explicable, dado que hogares numerosos con bajo ingreso tienen menos probabilidades de garantizar la seguridad alimentaria de sus integrantes, que aquellos en igualdad de condiciones, pero que cuentan con un número menor de miembros. ${ }^{19}$

La escala presentó una asociación estadísticamente significativa con la ocupación del padre y el jefe del hogar que, por sus características socioeconómicas, eran de bajo perfil y remuneración. Esta situación limita el acceso de los hogares a la cantidad y calidad de alimentos que requieren, así inviertan un alto porcentaje de sus ingresos en la compra de alimentos. ${ }^{2}$

\section{Conclusión}

Los resultados de este estudio indican que la escala de percepción de seguridad alimentaria en hogares con niños menores de 10 años, adaptada y validada en hogares rurales y urbanos en Antioquia, Colombia, es un instrumento válido para identificar la magnitud y gravedad de la inseguridad alimentaria en esa región.

\section{Agradecimientos}

Los investigadores agradecen a la Gobernación de Antioquia, la Dirección Seccional de Salud de Antioquia y al Plan de Mejoramiento Alimentario y Nutricional de Antioquía (MANA), que participaron en la financiación del estudio; a la Universidad de Antioquia y la Escuela de Nutrición Dietética, que contribuyeron con recursos financieros, humanos y tecnológicos para su desarrollo, y a los hogares por haber compartido con nosotros un aspecto importante de su vida cotidiana.

\section{Referencias}

I. Organización de las Naciones Unidas para la Agricultura y la Alimentación. Declaración de Roma sobre la seguridad alimentaria mundial y plan de Acción de la Cumbre Mundial. Documento técnico de referencia. Roma: FAO, 1996. Disponible en www.fao.org/index_es.htm. 2. Organización de las Naciones Unidas para la Agricultura y la Alimentación. El estado de la inseguridad alimentaria en el mundo. Seguimiento de los avances en la consecución de los objetivos de la Cumbre Mundial sobre la Alimentación y de los Objetivos de desarrollo del Milenio. Roma: FAO, 2004. Disponible en www.fao.org/docrep/fao/ 007/y5650s/y5650s00.pdf.

3. Organización de las Naciones Unidas para la Agricultura y la Alimentación. Sexta Encuesta Alimentaria Mundial.Viale Terne di Caracalla. Roma: FAO, 1996.

4. Organización de las Naciones Unidas para la Agricultura y la Alimentación. Medición y evaluación de la carencia de alimentos y la desnutrición. Resumen de orientación. Simposio Científico Internacional. Roma: FAO, 2002. Disponible en http://www.fao.org/ DOCREP/005/Y4250S/Y4250S00.HTM.

5. Radimer KL. Measurement of Household Food Security in the USA and other Industrialized Countries. Public Health Nutr 2002;5:859-864. 6. Wehler CA, Scott RI, Anderson J]. The Community Childhood Identification Proyect:A Model of Domestic Hunger-Demonstration Project in Seattle, Washington. J Nutr Ed 1992;24:29S-35S.

7. Frongillo EA.Validation of Measures of Food Insecurity and Hunger.J Nutr 1999;129(2S Suppl):506S-509S.

8. Gary B, Nord M, Price C, Hamilton W, Cook J. Guide to Measuring Household Food Security, Revised 2000.Alexandria VA: U.S. Department of Agriculture, Food and Nutrition Service, 2000.

9. Lorenzana P, Danjur D. La adaptación y validación de una escala de seguridad alimentaria en una comunidad de Caracas, Venezuela. Arch Latinoamer Nutr 2000;50(4):334-340.

10. Melgar-Quiñonez H, Kaiser L, Martin A, Metz D, Olivares A Inseguridad alimentaria en latinos de California: observaciones de grupos focales. Salud Publica Mex 2003;45(3): 198-205.

I I. Melgar-Quiñonez H, Zubieta AC,Valdez E, Whitelaw B, Kaiser L. Validación de un instrumento para vigilar la inseguridad alimentaria en la Sierra de Manantlán, Jalisco. Salud Publica Mex 2005;47(6):4I3-422. 
12. Fiszbein A, Giovagnoli PI. Hambre en Argentina. Oficina Banco Mundial para la Argentina, Chile, Paraguay y Uruguay. Buenos Aires: 2003; Documento de Trabajo $N^{\circ}$ 4/03. Disponible en: www.banco mundial.org.ar.

13. Pérez-Escamilla R, Correa-Segall A, Kurdian L,Archanjo M, Marín-León L, Panigassi G. An Adapted Version of the U.S. Department of Agriculture Food Insecurity Module Is a Valid Tool for Assessing Household Food Insecurity in Campinas, Brazil. J Nutr 2004; 134: 1923-1928.

14. Bond TG, Fox CM. Applying the Rasch Model: Fundamental Measurement in the Human Sciences. New Jersey: Lawrence Erlbaum Associates, 200I.

15. Castaño E. Cuantificación de variables cualitativas y análisis de componentes principales. Simposio de Estadística y Análisis Multivariado de Datos. Bogotá: Universidad Nacional de Colombia, 1994:I-22.
16. Lorenzana-Albert P. Seguridad alimentaria, tecnología y nutrición. Agroalimentaria 1999(8):49-57.

17. Mercado-Suárez CE, Lorenzana-Albert P.Acceso y disponibilidad alimentaria familiar.Validación de instrumentos para su medición. Caracas: Refolit, 2000

18. Figueroa D. Seguridad alimentaria familiar. Rev Salud Pública Nutr 2003;4(2).

19. Guevara DA. Dinámica de la pobreza en Colombia: análisis de los ingresos de jefes de hogar urbano 1984-2003. Rev Econ y Des 2005;2(4):77-108. 